\title{
A NEW QCD ANALYSIS OF POLARIZED DIS
}

\section{E. Leader*}

Imperial College, University of London, London WC1E 7HX, England E-mail:

\section{A.V. Sidorov ${ }^{\dagger}$}

Bogoliubov Theoretical Laboratory, JINR, Dubna, Russia

E-mail: 'sidorovothsun1.jinr.rui

\section{D.B. Stamenov}

Institute for Nuclear Research and Nuclear Energy, Sofia, Bulgaria

E-mail: 'stamenov@inrne.bas.bgi

Abstract: We present a new next-to-leading order (NLO) QCD analysis of the world data on inclusive polarized deep inelastic scattering. A new set of polarized parton densities is extracted from the data and the sensitivity of the results to different positivity constraints is discussed.

\section{Introduction}

Deep inelastic scattering (DIS) of leptons on nucleons has remained the prime source of our understanding of the internal partonic structure of the nucleon and one of the key areas for the testing of perturbative QCD. Decades of experiments on unpolarized targets have led to a rather precise determination of the unpolarized parton densities. Spurred on by the famous EMC experiment [1] at CERN in 1988, there has been a huge growth of interest in polarized DIS experiments which yield more refined information about the partonic structure of the nucleon, i.e. how the nucleon spin is divided up among its constituents, quarks and gluons. Many experiments have been carried out at SLAC, CERN and DESY. In this talk we present an updated version of our NLO polarized parton densities (PD)

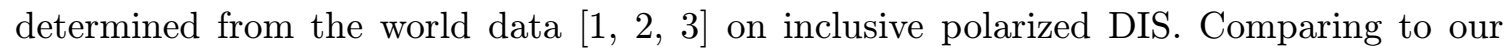
previous analysis $\left[\begin{array}{l}\overline{4} \\ \mathbf{1}\end{array}\right]$ :

${ }^{*}$ This research was supported by the UK Royal Society and the JINR-Bulgaria Collaborative Grants, by the RFBR (No 00-02-16696), INTAS 2000 (No 587) and by the Bulgarian National Science Foundation under Contract 1010.

${ }^{\dagger}$ Speaker. 
i) For the axial charges $g_{A}$ and $a_{8}$ their updated values are used:

$$
g_{A}=\mathrm{F}+\mathrm{D}=1.2670 \pm 0.0035, \quad \mathrm{a}_{8}=3 \mathrm{~F}-\mathrm{D}=0.585 \pm 0.025 .
$$

ii) In our ansatz for the input polarized PD

$$
\Delta f_{i}\left(x, Q_{0}^{2}\right)=A_{i} x^{\alpha_{i}} f_{i}^{\mathrm{MRST}}\left(x, Q_{0}^{2}\right)
$$

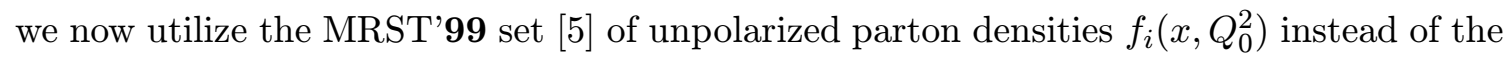
MRST'98 one. In (1'1.2') $A_{i}, \alpha_{i}$ are free parameters (6 parameters in our fit after using the sum rules (1, $\left.\overline{1}_{1} \overline{1}_{1}\right)$ for the quark polarizations).

iii) The recent SLAC/E155 proton data [30] 30 are incorporated in the analysis.

iv) The positivity constraints on the polarized PD are discussed.

\section{Method of Analysis}

The spin-dependent structure function of interest, $g_{1}^{N}\left(x, Q^{2}\right)$, is a linear combination of the asymmetries $A_{\|}^{N}$ and $A_{\perp}^{N}$ (or the related virtual photon-nucleon asymmetries $A_{1,2}^{N}$ ) measured with the target polarized longitudinally or perpendicular to the lepton beam, respectively. Neglecting as usual the sub-dominant contributions, $A_{1}^{N}\left(x, Q^{2}\right)$ can be expressed via the polarized structure function $g_{1}^{N}\left(x, Q^{2}\right)$ as

$$
A_{1}^{N}\left(x, Q^{2}\right) \cong\left(1+\gamma^{2}\right) \frac{g_{1}^{N}\left(x, Q^{2}\right)}{F_{1}^{N}\left(x, Q^{2}\right)}, \quad N=p, n, d,
$$

where $F_{1}^{N}$ is the unpolarized structure function and $\gamma^{2}$ is a kinematic factor.

In the NLO QCD approximation the quark-parton decomposition of $g_{1}\left(x, Q^{2}\right)$ has the following form:

$$
g_{1}\left(x, Q^{2}\right)=\frac{1}{2} \sum_{q}^{N_{f}} e_{q}^{2}\left[(\Delta q+\Delta \bar{q}) \otimes\left(1+\frac{\alpha_{s}\left(Q^{2}\right)}{2 \pi} \delta C_{q}\right)+\frac{\alpha_{s}\left(Q^{2}\right)}{2 \pi} \Delta G \otimes \delta C_{G}\right],
$$

where $\Delta q\left(x, Q^{2}\right), \Delta \bar{q}\left(x, Q^{2}\right)$ and $\Delta G\left(x, Q^{2}\right)$ are quark, anti-quark and gluon polarized densities which evolve in $Q^{2}$ according to the spin-dependent NLO DGLAP equations. $\delta C_{q, G}$ are the NLO terms in the spin-dependent Wilson coefficient functions and the symbol $\otimes$ denotes the usual convolution in Bjorken $x$ space. $\mathrm{N}_{\mathrm{f}}$ is the number of flavours.

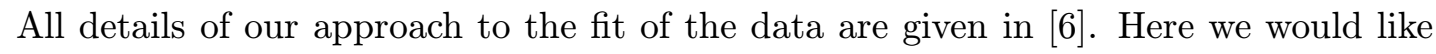
to emphasize only that according to this approach the NLO QCD predictions have been confronted to the data on the spin asymmetry $A_{1}^{N}\left(x, Q^{2}\right)$, rather than on the $g_{1}^{N}\left(x, Q^{2}\right)$. The choice of $A_{1}^{N}$ should minimize the higher twist contributions which are expected to partly cancel in the ratio $\left(\overline{2} . \overline{1}^{\prime}\right)$, allowing use of data at lower $Q^{2}$ (in polarized DIS most of the small $x$ experimental data points are at low $Q^{2}$ ). Indeed, we have found [i, i] that if for $g_{1}$ and $F_{1}$ leading-twist QCD expressions are used, the higher twist corrections $h(x)$ to $A_{1}\left(x, Q^{2}\right)=A_{1}^{Q C D}\left(x, Q^{2}\right)+h(x) / Q^{2}$ are negligible and consistent with zero within the

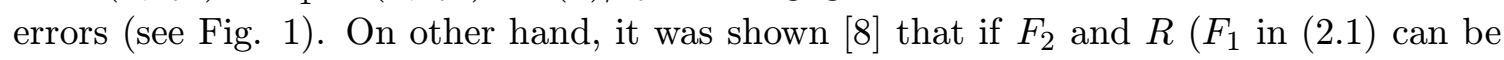


expressed via usually measured $F_{2}$ and $R$ ) are taken from experiment (as has been done in many analyses) the HT corrections to $A_{1}$ are sizeable and important. So, in order to analyze the $g_{1}$ data, the HT contribution to $g_{1}$ (unknown at present) has to be included in the analysis. These results clearly demonstrate the advantage of the approach used in our analysis.

Following the procedure of our previous analyses we have extracted the NLO (as well as LO) polarized parton densities from the fit to the world data on $A_{1}^{N}\left(x, Q^{2}\right)$ using for the flavour-nonsinglet combinations of their first moments

$$
\begin{gathered}
a_{3}=(\Delta u+\Delta \bar{u})\left(Q^{2}\right)-(\Delta d+\Delta \bar{d})\left(Q^{2}\right), \\
a_{8}=(\Delta u+\Delta \bar{u})\left(Q^{2}\right)+(\Delta d+\Delta \bar{d})\left(Q^{2}\right)-2(\Delta s+\Delta \bar{s})\left(Q^{2}\right),
\end{gathered}
$$

the sum rules (i.i. to the deviation of $a_{8}$ from its $\mathrm{SU}(3)$ flavour symmetric value (0.58) has been studied and the results are given in [i] $\left.\overline{1}_{0}\right]$. Here we will present only the polarized parton densities corresponding to the $\mathrm{SU}(3)$ symmetric value of $a_{8}$ in (1) (1)

What we can deduce from inclusive DIS in the ab-

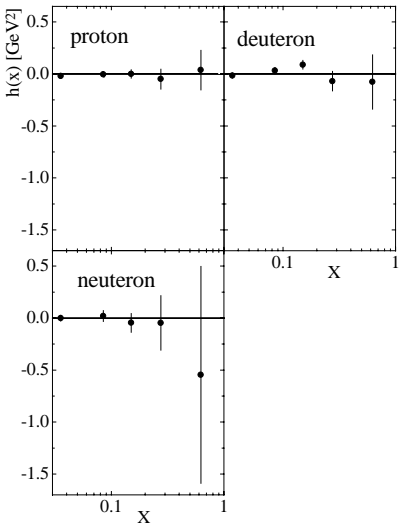

Figure 1: Higher twist contribution $h^{N}(x)$ to the spin asymmetry $A_{1}^{N}\left(x, Q^{2}\right)$ extracted from the data. sence of charged current neutrino data is the sum of the polarized quark and anti-quark densities

$$
(\Delta u+\Delta \bar{u})\left(x, Q^{2}\right), \quad(\Delta d+\Delta \bar{d})\left(x, Q^{2}\right), \quad(\Delta s+\Delta \bar{s})\left(x, Q^{2}\right)
$$

and the polarized gluon density $\Delta G\left(x, Q^{2}\right)$. The non-strange polarized sea-quark densities $\Delta \bar{u}\left(x, Q^{2}\right)$ and $\Delta \bar{d}\left(x, Q^{2}\right)$, as well as the valence quark densities $\Delta u_{v}\left(x, Q^{2}\right)$ and $\Delta d_{v}\left(x, Q^{2}\right)$, cannot be determined without additional assumptions about the flavour decomposition of the sea. Nonetheless (because of the universality of the parton densities) they are of interest for predicting the behaviour of other processes, like polarized $p p$ reactions, etc. That is why, we extract from the data the PD $(2.5)$ and $\Delta G\left(x, Q^{2}\right)$, as well as the valence parts $\Delta u_{v}\left(x, Q^{2}\right)$ and $\Delta d_{v}\left(x, Q^{2}\right)$ corresponding to the assumption on the flavour symmetric sea $(\Delta \bar{u}=\Delta \bar{d}=\Delta \bar{s})$.

\section{Results}

The results of analysis are presented in the JET scheme $[\overline{9}]$. A remarkable property of the JET [and Adler-Bardeen (AB)] schemes is that the singlet $\Delta \Sigma\left(Q^{2}\right)$, as well as the strange sea polarization $\Delta s\left(Q^{2}\right)$, are $Q^{2}$ independent quantities. Then, in these schemes it is meaningful to directly interpret $\Delta \Sigma$ as the contribution of the quark spins to the nucleon spin and to compare its value obtained from DIS region with the predictions of the different (constituent, chiral, etc.) quark models at low $Q^{2}$. 
It is important to mention that the difference between the values of the strange sea polarization, obtained in the $\overline{\mathrm{MS}}$ and JET schemes could be large due to the axial anomaly.To illustrate how large it can be, we present the values of $(\Delta s+\Delta \bar{s})$ at $Q^{2}=1 \mathrm{GeV}^{2}$ obtained in our analysis of the world DIS data in the $\overline{\mathrm{MS}}$ and JET schemes $(\Delta G=0.68)$ :

$$
\begin{aligned}
(\Delta s+\Delta \bar{s})_{\overline{\mathrm{MS}}} & =-0.13 \pm 0.03 \\
(\Delta s+\Delta \bar{s})_{\mathrm{JET}} & =-0.07 \pm 0.01
\end{aligned}
$$

Note that if $\Delta G$ is larger than $0.68,(\Delta s+\Delta \bar{s})$ JET could vanish in agreement with what is intuitively expected in quark models at low- $Q^{2}$ region $\left(Q^{2} \approx 0\right)$.

As in our previous analysis a very good description of the world data on $A_{1}^{N}$ and $g_{1}^{N}$ is achieved (for the best fit $\chi^{2}=155.9$ for $\left.179 \mathrm{DOF}\right)$. The new theoretical curves for $A_{1}$ and $g_{1}$ corresponding to the best fit practically coincide

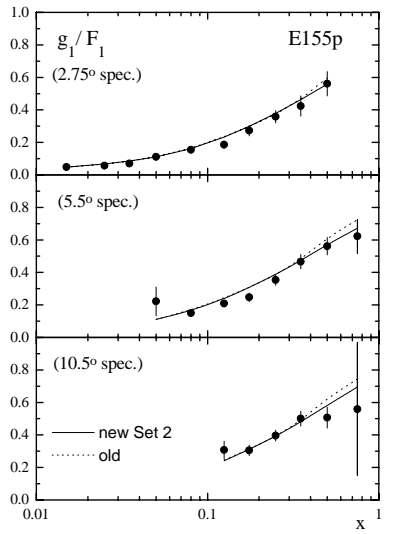

Figure 2: Comparison of our NLO result for $A_{1}^{p}$ with SLAC/E155p experimental data. Error bars represent the total errors. with the old ones. The agreement with the SLAC/E155p data is also very good (Fig. 2).

We have determined from the data two sets of polarized PD: i) Set1 - without any constraints on the polarized PD during the fit, ii) Set2 - fit to the data with imposition of LO positivity constraints determined by the MRST unpolarized densities. All polarized PD of Set1, with the exception of the strange sea density $\Delta s(x)$, are compatible with the LO positivity bounds imposed by the MRST unpolarized PD. However, if one uses the more accurate LO positivity bounds on $\Delta s(x)$ obtained by using the unpolarized strange sea density $s(x)_{B P Z}$ (Barone et al. [i] 1 in 1 ), the Set $\Delta s(x)$ lies in the allowed region. It is important to mention that $s(x)_{B P Z}$ is determined with a higher accuracy compared to other global fits. The Set1 PD are found to be within the error bands of the old PD, while the changes of the Set2 $(\Delta s+\Delta \bar{s})(x)$ and $\Delta G(x)$ are larger, especially for the strange sea quarks (Fig. 3 and 4 ).

Finally, let us summarize the changes in the quark and gluon polarizations (the first moments of PD) at $Q^{2}=1 \mathrm{GeV}^{2}$ which arise from the new analysis:

- $(\boldsymbol{\Delta} \mathbf{u}+\boldsymbol{\Delta} \overline{\mathbf{u}})$ and $(\boldsymbol{\Delta} \mathbf{d}+\boldsymbol{\Delta} \overline{\mathbf{d}})$ practically do not change.

- the magnitude of $|\boldsymbol{\Delta} \mathbf{s}+\boldsymbol{\Delta} \overline{\mathbf{s}}|$ has increased a little: $0.06 \pm 0.01($ old $) \rightarrow 0.07 \pm 0.01($ Set1 $) \rightarrow 0.09 \pm 0.02($ Set2)

- The central value of $\boldsymbol{\Delta} \boldsymbol{\Sigma}$ has decreased: $0.40 \pm 0.04($ old $) \rightarrow 0.37 \pm 0.04($ Set1 $) \rightarrow 0.32 \pm 0.05($ Set2)

- The axial charge $\mathbf{a}_{\mathbf{0}}\left(\mathbf{Q}^{2}\right)=\Delta \Sigma_{\overline{\mathrm{MS}}}\left(Q^{2}\right)=\Delta \Sigma_{\mathrm{JET}}-N_{f} \frac{\alpha_{s}\left(Q^{2}\right)}{2 \pi} \Delta G\left(Q^{2}\right)$ is also decreasing: $0.26 \pm 0.05($ old $) \rightarrow 0.23 \pm 0.06($ Set2 $) \rightarrow 0.21 \pm 0.06($ Set1 $)$

- For the gluon polarization $\Delta \mathbf{G}$ we have found that the positive values of $\Delta G$ lie in the wide range $[0,1.5]$ if one takes into account the correlations and the sensitivity 


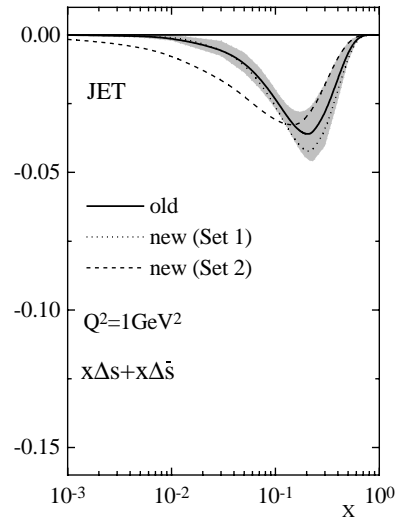

Figure 3: The polarized NLO strange quark sea density at $Q^{2}=1 \mathrm{GeV}^{2}$. The error bands account for the experimental uncertainties.

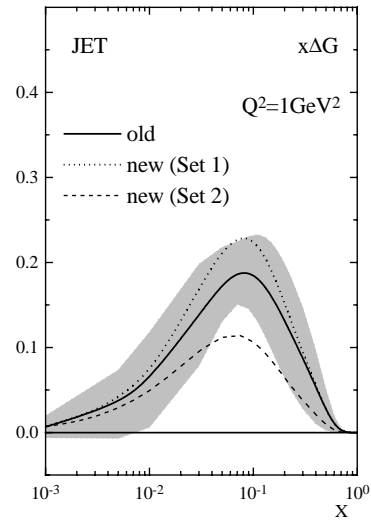

Figure 4: The polarized NLO gluon density at $Q^{2}=1 \mathrm{GeV}^{2}$. The error bands account for the experimental uncertainties.

to the $\mathrm{SU}(3)$ flavour symmetry breaking. A negative $\Delta G$ is still not excluded from the present DIS inclusive data.

\section{References}

[1] EMC, J. Ashman et al., Physs. Lett. B 206 (1988) 364

[2] SLAC E142 Coll., P.L. Anthony et al., 'Phys. Rev. D 54 (1997) $5520 ;$;LAC/E154 Coll., K. Abe et al., 'Phys. Rev. Lett. 79-1997)_26 SLAC E143 Coll., K. Abe et al., 'Phys. Rev. D 58

:

D. Ádeva et al., 'P hys. Rev. D $5 \overline{8}(\overline{1} 9 \overline{9}) \overline{1} 1200 \overline{1}$; HERMES Coll., K. Áckerstaff et al., 'Phys' (

[3] SLAC/E155 Coll., P.L. Anthony et al., 'Phys.Lett. B

[4] E. Leader, A.V. Sidorov, D.B. Stamenov, 'Phys. Lett. B $\mathbf{4} 62$ 2 $(1999) 1899_{1}^{\prime}$

[5] A.D. Martin, R.G. Roberts, W.J. Stirling and R.S. Thorne, 'Eur. Phys. J. 140000$) 1331$.

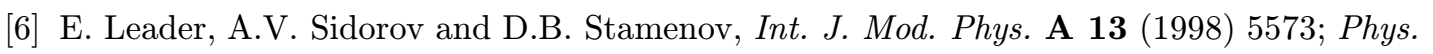
( Rev. D 58 (1998) 114028 .

[7] E. Leader, A.V. Sidorov and D.B. Stamenov, in Particle Physics at the Start of the New Millennium, edited by A.I. Studenikin, World Scientific, Singapore, 2001, pp 76-84 (Proc. of the 9th Lomonosov Conference on Elementary Particle Physics, Moscow, 1999).

[8] M. Glück. E. Reya, M. Stratmann and W. Vogelsang, 'Phys. Rev. D 63. 2001 094005.

[9] R. D. Carlitz, J. C. Collins and A.H. Mueller, Phys. Lett. B_214 (1988) 229'; M. Anselmino, A. V. Efremov and E. Leader, $\bar{P} \bar{h} \bar{y}$. Rept. $\mathbf{2} \mathbf{6} \overline{1}(\overline{1} 9 \overline{9})$ li. ; D. Müller and O. V. Teryaev, 'Phys.Rev. D $\overline{5} \mathbf{6}(\overline{1} \overline{9} \overline{7})-260 \overline{7}$

[10] E. Leader, A.V. Sidorov and D.B. Stamenov, Physs. Lett.

[11] V. Barone, C. Pascaud and F. Zomer, 'Eur. Phys. J. C 12 $(2000) 243$. 